\title{
A WORLDWIDE 3D GCP DATABASE INHERITED FROM 20 YEARS OF MASSIVE MULTI-SATELLITE OBSERVATIONS
}

\author{
L. Chandelier ${ }^{1 *}$, L. Coeurdevey ${ }^{2}$, S. Bosch ${ }^{2}$, P. Favé ${ }^{1}$, R. Gachet ${ }^{1}$, A. Orsoni ${ }^{1,}$, T. Tilak ${ }^{1}$, A. Barot ${ }^{2}$, \\ ${ }^{1}$ IGN, 6 avenue de l'Europe, 31520 Ramonville, France - \\ (laure.chandelier, pascal.fave, roland.gachet, alain.orsoni, thomas.tilak)@ign.fr \\ ${ }^{2}$ Airbus Defence and Space, 5 rue des Satellites, BP 14359, 31030 Toulouse Cedex 4, France - \\ (laurent.coeurdevey, sebastien.bosch, alexis.barot)@airbus.com
}

Commission TCII/1

KEY WORDS: Ground control point, Space reference point, SPOT 6/7, SPOT 5 HRS, registration, massive image processing, cloud computing

\begin{abstract}
:
High location accuracy is a major requirement for satellite image users. Target performance is usually achieved thanks to either specific on-board satellite equipment or an auxiliary registration reference dataset. Both methods may be expensive and with certain limitations in terms of performance. The Institut national de l'information géographique et forestière (IGN) and Airbus Defence and Space (ADS) have worked together for almost 20 years, to build reference data for improving image location using multi-satellite observations. The first geometric foundation created has mainly used SPOT 5 High Resolution Stereoscopic (HRS) imagery, ancillary Ground Control Points (GCP) and Very High Resolution (VHR) imagery, providing a homogenous location accuracy of $10 \mathrm{~m}$ CE90 almost all over the world in 2010.

Space Reference Points (SRP) is a new worldwide 3D GCP database, built from a plethoric SPOT 6/7 multi-view archive, largely automatically processed, with cloud-based technologies. SRP aims at providing a systematic and reliable solution for image location (Unmanned Aerial Vehicle, VHR satellite imagery, High Altitudes Pseudo-Satellite...) and similar topics thanks to a high-density point distribution with a $3 \mathrm{~m}$ CE90 accuracy.

This paper describes the principle of SRP generation and presents the first validation results. A SPOT 6/7 smart image selection is performed to keep only relevant images for SRP purpose. The location of these SPOT 6/7 images is refined thanks to a spatiotriangulation on the worldwide geometric foundation, itself improved where needed. Points making up the future SRP database are afterward extracted thanks to classical feature detection algorithms and with respect to the expected density. Different filtering methods are applied to keep the best candidates. The last step of the processing chain is the formatting of the data to the delivery format, including metadata. An example of validation of SRP concept and specification on two tests sites (Spain and China) is then given. As a conclusion, the on-going production is shortly presented.
\end{abstract}

\section{INTRODUCTION}

A large part of the Earth Observation market (EO) anticipates better image resolution, better accuracy, cost saving platforms and massive processing. Imagery location accuracy, and subsequently horizontal performance for orthoimages, is a key requirement for users. This request increases dramatically with the data resolution, needs of geometrically consistent images for time series analysis, addressing various scales, from local with aerial data to global with satellite data. For very high resolution, a location standard of $5 \mathrm{~m}$ is emerging, potentially more demanding in the future.

\section{NEED FOR NEW GENERATION OF REGISTRATION DATABASE}

\subsection{Context}

The coming Pléiades Neo new constellation (Airbus, 2020) of optical satellites with a $30 \mathrm{~cm}$ resolution shall fulfil the location accuracy requirement better than $5 \mathrm{~m}$ at sensor level. Pléiades Neo also intends to achieve a planimetric accuracy on moderate slopes $(<20 \%)$ of $5 \mathrm{~m} \mathrm{CE90} \mathrm{for} \mathrm{standard} \mathrm{orthoimagery}$ rectified with high quality DSM/DTM, such as Airbus WorldDEM4Ortho (Airbus, 2020-b). As acquisition angle effects coupled with Earth relief are not perfectly compensated worldwide by the DSM/DTM, the adequate geometric sensor model should target accuracy of around $3 \mathrm{~m}$, that is to say, up to $2 \mathrm{~m}$ better than the satellite native location requirement.

Ensuring native high location accuracy on satellite involves extremely expensive on-board equipment (gyrometer, star tracker, etc.) as well as significant monitoring costs for calibration tasks. Despite their sophistication, a stable location performance of around $4 \mathrm{~m}$ (a few micro radians) is the current limit nearly achieved with on-board equipment. Such a design also generates a risk since a failure of this equipment would have a critical impact on the mission. Exceeding this performance and/or costs means on-ground geometric model refining, possibly by image processing. Fully automatic refining processing is expected by image matching on large reference data of higher accuracy. Global registration databases are designed to fulfil this refining processing.

\subsection{Example of existing global registration databases}

This section presents global approaches - worldwide coverage in the satellite imagery case.

\subsubsection{Reference registration layers}

This type of registration database is composed of a continuous orthoimage layer associated with a DSM.

\footnotetext{
* Corresponding author
} 
As from 2002, SPOT Image S.A. Company Toulouse, France, now part of Airbus Group, and IGN (Institut national de l'information géographique et forestière), co-developed the concept of reference layers to produce the 3D worldwide database called Reference3DC) (SPOT Image, 2011). Reference3D(C) contains a dual endogenous layer: a horizontal layer of continuous $5 \mathrm{~m}$ optical orthoimages and a vertical layer with a 30m DSM (DTED2), both delivered with associated quality data. Source data are composed of SPOT 5 High Resolution Stereoscopic (HRS) instrument with an extreme geometric rigidity, processed through continental block triangulation over $80 \mathrm{Mkm}^{2}$. The database was upgraded several times with SPOT 5 High Resolution Geometric (HRG) then SPOT 6/7 orthoimages, and enlarged to continuously cover more than $110 \mathrm{Mkm}^{2}$. Today designated as Premium Accuracy Services (PAS), it provides a global accuracy for orthoimages of 6-8m CE90 horizontally and 5-10m LE90 vertically on moderate slopes $(<20 \%)$, see Figure 1 .

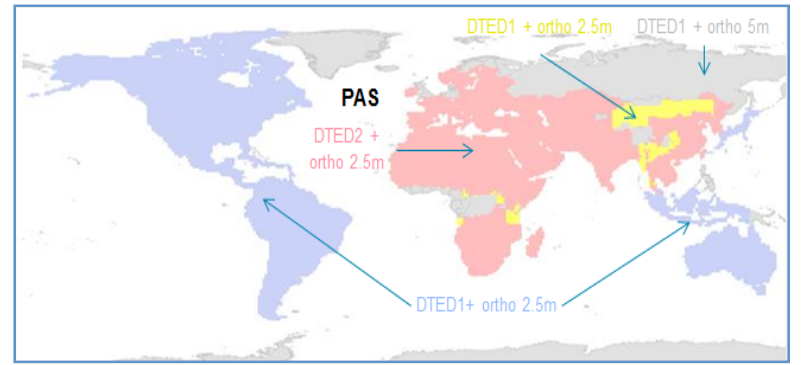

Figure 1. Airbus/IGN PAS dual layers database coverage. The area in pink also depicts the IGN BDAmer database coverage.

The fundamental advantage of the PAS dual layers concept is to allow a straightforward use of massive automatic image algorithms such as matching registration or orthoimages rectified with the DSM layer. The SPOT Image service Ortho On Line (2010) demonstrated its ability to manage multi-sensor destination images.

The disadvantage of these reference registration layers is that the 3D coordinates are not constructed with a perfect consistency, due to potential differences in resolution, date and altitude considered (surface vs terrain) between DSM and orthoimage layers. These gaps will limit target accuracy under $5 \mathrm{~m}$. Moreover, the matching process can be complicated when no upstream pre-selection of relevant GCP has been conducted.

2.2.2 3D feature points extracted from images with a high accuracy location

This type of reference database uses the possibility to extract 3D GCP with image chips, nearly as much as necessary, from any feature points taken on existing "well-located" images. This concept was first released in 2009 with the BDAmer GCP database (from the French word "amer", ie landmark). BDAmer is derived from the underlying SPOT 5 HRS worldwide geometric foundation. Both are described in section 3.2. The 3D coordinates of the BDAmer GCP are perfectly consistent, but the density is not high enough for individual small swath image registration. The 5m GSD (Ground sample distance) of the chips and their content, which dates back to the 2000's, do not fit with the next decade's Very-High-Resolution (VHR) satellite imagery either.

\subsection{The SRP initiative}

The Space Reference Point (SRP) initiative was first introduced in 2017 to challenge a $3 \mathrm{~m}$ location performance worldwide for the new Airbus' Pléiades Neo constellation, through fully massive on-ground registration processing with no human interactions. As explained previously, achieving such an accuracy level will not be possible with a continuous orthoimage layer associated with a DSM. SRP is based on the more promising 3D feature points approach, as a successor of BDAmer.

SRP is a global 3D GCP database built from SPOT 6/7 images whose registration is mainly refined on a worldwide stereoscopic HRS geometric foundation. With respect to the successive improvements made during twenty years by Airbus and IGN in theirs co-works, the geometric foundation has proved that a high accuracy can be achieved worldwide. GCP image chips are extracted from the source image archive.

More generally, SRP is expected to register single images on small areas of interest of up to $30 \mathrm{~km}^{2}$, with decimetre resolutions. The aim is also to encompass emerging use cases of poor native deregistration of up to $150 \mathrm{~m}$ as seen with small or low-cost satellite constellations, High Altitudes Pseudo-Satellite (HAPS) or Unmanned aerial vehicles (UAV) images. Various 3D imagery applications can also be considered.

Airbus and IGN have benefitted from three opportunities to propose this innovative solution:

- In replacement of SPOT 5 images, the plethoric SPOT 6/7 image archive to ensure better resolution, freshness and exhaustive multi-view source images all over the world for stereo computation.

- The improvement of the HRS geometric foundation and its BDAmer derivative with a performance better than $3 \mathrm{~m}$ over the full land mass thanks to external data.

- The massive computing on cloud infrastructure for production tasks and time planning.

The SRP method is designed to get GCP with:

- A qualified accuracy in 3D obtained thanks to a space intersection of the corresponding rays.

- A potentially high density with respect to the landscape.

- A resolution adapted to the target imagery.

- A choice of appropriate perennial features.

- A sizeable coverage nearly everywhere.

- A capability to register images with poor native location.

The main requirements of the SRP database are the following:

\begin{tabular}{|lcl|}
\hline Coverage & $\begin{array}{c}\text { Requirement } \\
\text { Entire Earth land } \\
\text { surface }(\text { around } \\
\left.135 \mathrm{Mm}^{2}\right)\end{array}$ & $\begin{array}{l}\text { Exclude Antarctic, } \\
\text { Greenland and large ice } \\
\text { fields. Include islands. }\end{array}$ \\
\hline $\begin{array}{l}\text { GCP } \\
\text { density }\end{array}$ & $\begin{array}{c}1 \mathrm{GCP} / 2 \mathrm{~km}^{2} \text { in } \\
\text { average upon } 1 / 100 \\
\text { square degree }\end{array}$ & $\begin{array}{l}\text { Possibly released on } \\
\text { remote areas. }\end{array}$ \\
\hline $\begin{array}{l}\text { GCP } \\
\text { accuracy }\end{array}$ & $3 \mathrm{~m} \mathrm{CE90}$ & $\begin{array}{l}\text { Possibly released on } \\
\text { complex landscapes (huge } \\
\text { mountain ranges, remote } \\
\text { islands, etc.) }\end{array}$ \\
\hline
\end{tabular}

Table 1: SRP main requirements 
The SRP production began in March 2018, with a capacity of $3 \mathrm{Mkm}^{2}$ per month, set to be ready over the "useful" Earth land surface (around $109 \mathrm{Mkm}^{2}$ ) at the end of 2020. Siberia, Northern parts of North America and remote islands are anticipated for the end of 2021, at least a total of $135 \mathrm{Mkm}^{2}$.

\section{SRP PROCESSING CHAIN}

\subsection{Global workflow and architecture}

A specific workflow has been developed to produce the entire SRP database in three years. The different steps of the SRP processing chain are described in the next sections. The figure below shows the global workflow and the architecture involved. The first step, described in section 3.2, is the improvement of the worldwide geometric foundation. Simultaneously (see section 3.3), a smart selection of SPOT 6/7 on the entire archive is computed and then input to launch the image production itself. From these two input data, feature points are detected on a cloud platform (section 3.4) and used in a classical spatiotriangulation to refine the SPOT 6/7 image location and to keep best SRP candidates (section 3.5). At the end (section 3.6), SRP product is built from the most reliable points.

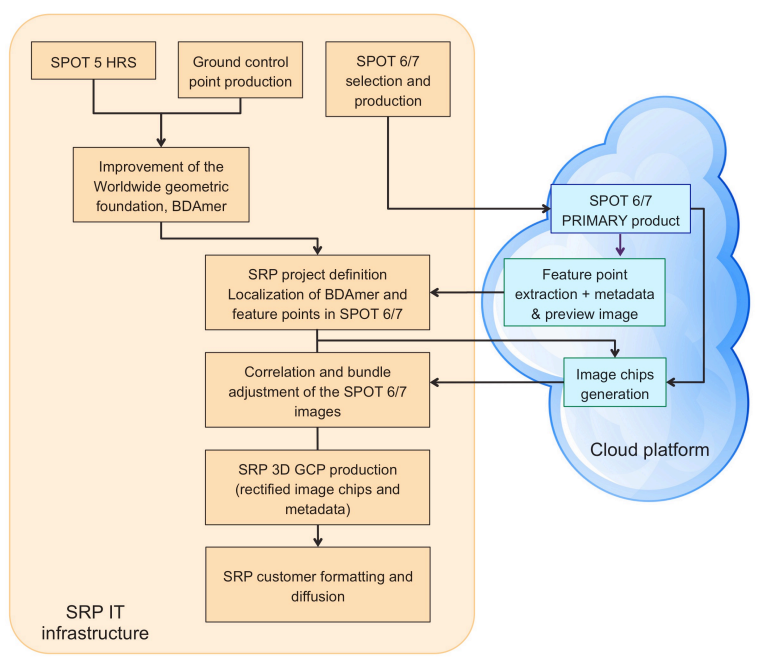

Figure 2. SRP workflow

Since the global amount of data to be processed in the workflow is at the petabyte scale, many optimisations were carried out to reach a reasonable production time. Thus, some operations are directly performed on a cloud infrastructure. Moreover, the worldwide production is divided into continental batches in order to deliver the product gradually. Each batch is made up of tens of thousands of images. A batch is a continuous block of SPOT 6/7 images designed to have borders in accordance with the landscape (to avoid borders between blocks in difficult areas such as mountains) and with an overlapping area between blocks.

\subsection{Improvement of the worldwide geometric foundation and its BDAmer}

The location accuracy is mainly founded on a worldwide stereo image bundle block adjustment with external GCP, called "geometric foundation" in the following sections.

\subsubsection{SPOT 5 HRS block}

The geometric foundation is mostly composed of SPOT 5 HRS images acquired between July 2002 and August 2014. HRS images are stereoscopic pairs with a base to height ratio of 0.8 . They have a swath of $120 \mathrm{~km}$ and a length of up to $600 \mathrm{~km}$ with a $5 \mathrm{mx} 10 \mathrm{~m}$ pixel. Location performance at the single pair level has been estimated at about $27 \mathrm{~m}$ at $90 \%$ (Bouillon et al., 2006) with no ground control points.

The benefit of building blocks with these wide and rigid stereoscopic strips was quickly demonstrated to improve location accuracy as well as global consistency between images. (Bouillon et al., 2006) shows that a bundle block adjustment helps achieve a location of better than $15 \mathrm{~m}$ at $90 \%$, without GCP. Blocks were expanded in order to improve location quality, to reach a more homogeneous location and to minimize geometrical deviations at block borders. In 2009, tie points were automatically measured through a feature detection algorithm to provide a homogeneous and controlled density of reliable tie points (a few hundred points per square degree). External $\mathrm{Z}$ reference (Shuttle Radar Topography Mission, SRTM and Ice, Cloud, and land Elevation Satellite, ICESat data) has also been added to the block in order to improve altimetry reliability or quality.

At the end of the 2000s, fine georeferencing becomes a central challenge for users who expect a location compliant with terrestrial positioning systems such as GPS. Consequently, external GCP were added to enhance the absolute location quality. (Breton et al., 2009) shows that the addition of GCP (approximately every $3,000 \mathrm{~km}$ ) and the computation of blocks of 1,000 images make it possible to reach a location close to $10 \mathrm{~m}$.

In 2013, 20,000 SPOT 5 HRS images were finally refined in a single block to ensure a unique and consequently coherent location all around the world. Increasing the size of the bundle adjustment blocks was possible thanks to a decrease in computation time, tuning of the different bundle adjustment settings (parameters and weighting), false point filtering and improvement of the point equipment (Massera et al., 2012). In order to reach the $3 \mathrm{~m} \mathrm{CE90} \mathrm{accuracy} \mathrm{all} \mathrm{over} \mathrm{the} \mathrm{world,} \mathrm{ground}$ control points are added locally to the worldwide geometric foundation.

\subsubsection{GCP equipment}

Numerous external GCP are needed to achieve the targeted accuracy for the SRP database. Over the years and with the technical system maturity level, the offer in terms of GCP diversifies and widens:

- Download of free available data: mainly permanent geodesic station networks and national orthoimage programs (as BDOrtho ${ }^{\circledR}$ for France).

- Purchase of GPS points provided by surveying companies.

- Very High Resolution independent group of $n$ image with native unbiased calibration around $\sigma_{\text {image }}=5 \mathrm{~m}$ (Geoeye, Pléiades, WorldView, possibly mixed) allow to build a "Ground control image" with an accuracy better than $\frac{\sigma_{\text {image }}}{\sqrt{n}}$ meters (Massera et al., 2012).

- Airbus Albatros airport database: these are Pléiades orthoimages adjusted in most cases on GPS GCP and rectified on a very fine endogenous DTM.

Others sources could be used as radar imagery GCP (TerraSAR$\mathrm{X})$ transferred to an optical image. This diversification makes it 
possible to obtain high location performances nearly all over the world. Figure 10 presents the current geometric foundation (SPOT 5 HRS imagery and GCP).

3.2.3 BD Amer: a GCP database which facilitates the use of the geometric HRS foundation

Beyond the exploitation of the HRS images themselves, the global HRS block may become a reference for adjusting location models for new images. But, the direct use of the HRS block for location adjustment is complex and difficult to manage.

Thus, for a long time, the location adjustment of new images was built with orthoimages (see section 2.1). The innovative BDAmer concept was born in 2009: BDAmer is a GCP database composed of homologous points extracted on HRS images and fully qualified. One strong advantage of this product is that every GCP is seen in stereoscopy (thanks to HRS instrument), sometimes with a higher multiplicity, and a long time interval. All GCP comes with $201 \times 201$ pixels multi-view SPOT 5 HRS chips. This database of 3D points quickly demonstrates its ability to automatically adjust the location of VHR images or to perform quality control (Gascon et al., 2017).

In the SRP processing chain, these BDAmer points are used as GCP to refine SPOT 6/7 model location and reach the expected accuracy.

\subsection{SPOT $6 / 7$ selection and production}

\subsubsection{SPOT $6 / 7$ archive}

SPOT 6/7 satellites are two commercial high-resolution optical imaging satellites, part of the Airbus Defence \& Space GEO constellation. They offer 4 multispectral bands (R,G,B,NIR) and $1.5 \mathrm{~m}$ resolution imagery in panchromatic mode over a large $60 \mathrm{~km}$ swath, and up to $600 \mathrm{~km}$ long. Thanks to their high agility, the daily acquisition capacity is up to $6 \mathrm{Mkm}^{2}$ when operating simultaneously.

Among a large satellite constellation, SPOT 6/7 has been chosen as the satellite data which is most adapted to SRP constraints: a large swath as per SPOT 5, a resolution rounded up to $2 \mathrm{~m}$ sufficient for precise image matching at $20 \mathrm{~cm}(1 / 10$ pixel), a good variety of acquisition angles for multi-temporal stereo observations, a plethoric image archive covering around 50 times the full land mass and acquired with strict monitoring of lowest cloud rate. Achieving this first SRP worldwide coverage with SPOT 6/7, it is possible that other satellite mission data may be input in the future.

The SPOT 6/7 archive contains more than $1.3 \mathrm{M}$ strips at the time this article is written. This archive spans a time range from 2013 to 2020, with SPOT 6 acquisitions beginning in 2013 and SPOT 7 in 2014. The acquisitions are done at a fully world scale, fulfilling Airbus' customers' specific needs. In parallel, a background task images each point of the globe at least once a year. Figure 3 gives a geographical overview of a subset of this image archive filtered with less than $15 \%$ of cloud cover.

Obviously, in some tropical areas there are no images with less than $15 \%$ cloud cover, yet there are some images with more clouds in the complete archive.

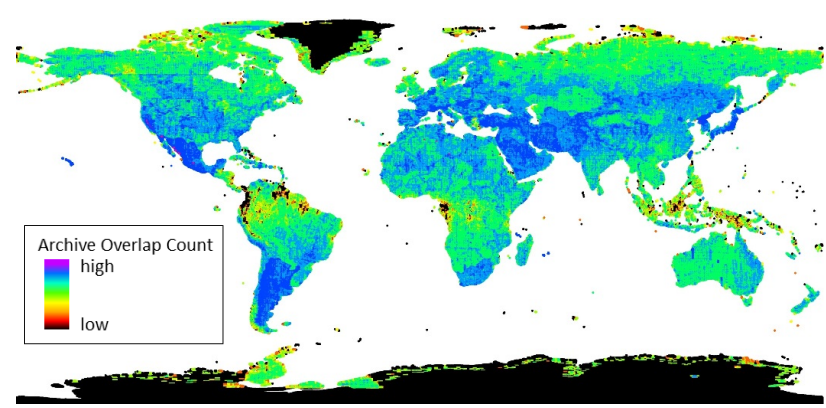

Figure 3. SPOT 6/7 image archive overlap count, from 2013 to mid-2019 with less than $15 \%$ of cloud cover over the image.

\subsubsection{Archive selection and production}

In order to compute the Space Reference Points, more than 400,000 strips (around 30\% of the whole SPOT 6/7 archive) are used. This can be compared with around 20,000 SPOT 5 HRS strips already input in the geometric foundation. This selection is carefully crafted in order to select the appropriate set of SPOT 6/7 with respect to the SRP production, but also to be minimal for obvious efficiency reasons. Therefore this selection must enforce these specific constraints:

- Selecting multi-stereo observations:

- Providing images with an overlap count exceeding 6 in the production area (usually a whole continent) and ensuring an adequate acquisition angle distribution.

- Limiting the overlap count to 30 images.

- Excluding unusable features:

- Taking into account the cloud mask in the overlap count.

- Limiting images with seasonal snow coverage.

- Along coastal areas, selecting images with sufficient land mass to facilitate the geometric processing.

- Avoiding redundant image selection, like ones sharing the same acquisition angle, season and year.

With these numerous constraints, carrying out such a selection at a worldwide scale required the design of specific tools in order to automate the process and to avoid every timeconsuming step involved in the choice of suitable images. A first "naïve" selection is done using all images with less than $15 \%$ cloud cover. Then this selection is enforced by the land mass constraint and the overlap count with respect to the cloud cover. In the areas with a weak overlap count, an iterative process relaxed the cloud cover constraint until the target overlap count is reached. Finally, redundant images are removed. Figure 4 illustrates the benefits of this method on the image selection for South America. An optimal set of images is selected while data efficiency is improved.

This optimization represents a $12 \%$ improvement in data efficiency while the selection of images is more homogenous and the gaps are almost filled (with the exception of a few extreme areas in terms of cloud coverage).

The selected images are then effectively produced in full resolution at sensor geometry level (PRIMARY), panchromatic and multispectral (bundle) with a normalized reflectance with respect to Rayleigh diffusion. The whole image production counts for more than 1 petabyte of data and coverage above $2,500 \mathrm{Mkm}^{2}$. Images have been processed at a very high rate and then uploaded in an object-store of the cloud platform for SRP processing. 


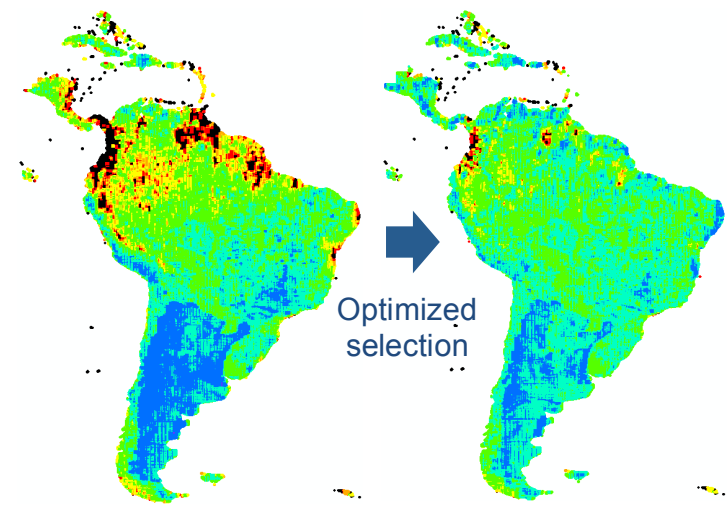

Figure 4. SPOT 6/7 optimized archive selection with respect to cloud cover and acquisition attributes. Left: initial selection with less than $15 \%$ cloud cover over the image. Right: optimized selection with a relaxed cloud cover constraint in low overlap count areas and redundant image removal. Colour range same as previous figure.

\subsection{Cloud processing for feature point extraction}

Two main processes run directly on the cloud platform on SPOT 6/7 PRIMARY products.

At the beginning of the processing chain, feature points candidating to GCP extraction are created thanks to a custommade highly scalable architecture.

Classical GCP, collected by survey or picked manually on the image, are often associated with on-ground paint mark, corner, intersection, thin pylon, etc. In contrast, the SRP GCP are image chips centred on feature points, computed with classical methods as maximum of Hessian value and representing high peaks of contrast, either bright (common case), or dark spots. They are particularly adapted to identification by image matching processing, rather than human identification, even if the resolution of the destination image is different to the GCP image chip. The density of the feature points is set higher than the SRP specification, and much higher than the bundle adjustment requirement. This large margin allows filtering of false points while keeping enough good points at the end of the process. A specific algorithm has been designed to reach a regular point distribution. Feature points positions are streamed on-the-fly from cloud storage to the SRP IT infrastructure. Other data, required for the following processing chain, come with the points: for each image, a subsampled preview by a factor of 10 (used to produce the overview chip) and various metadata such as the initial geometric model or the cloud mask.

As explained above, the full resolution images themselves remain on the cloud. To achieve the correlation process and the generation of the SRP image chips, only required image chips centred on feature point and GCP candidates are read from the cloud. This yields to a massive gain in external data transfer and therefore a huge impact on processing time and costs. The table below shows the amounts involved for the different types of data for the European batch and demonstrates the gain of this workflow organisation.

\begin{tabular}{|l|l|c|c|}
\hline \multicolumn{1}{|c|}{ Type of data } & \multicolumn{1}{|c|}{ Number } & Storage & $\begin{array}{c}\text { Size } \\
(\mathbf{T b})\end{array}$ \\
\hline PRIMARY SPOT imagery & $\approx 27,000$ images & Cloud & 91 \\
\hline Subsampled SPOT preview & $\approx 27,000$ images & SRP IT & 1,5 \\
\cline { 1 - 2 } $\begin{array}{l}\text { Feature point and GCP } \\
\text { infra- } \\
\text { ships }\end{array}$ & $10 \mathrm{M}$ points & 2,8 \\
${$\cline { 1 - 2 }$} }$ & $5 \mathrm{M}$ points & & 1,5 \\
\hline
\end{tabular}

Table 2: Size of the data for the European batch

\subsection{SPOT $6 / 7$ bundle block adjustment}

\subsubsection{Tie points}

Feature points are created on SPOT 6/7 imagery according to a priority criterion based on cloud coverage, season, freshness, low incidence angle. Ground position of these points is computed with the PRIMARY geometric model on the best available DSM. Then, image chips can be produced as described in part 3.4, and a conventional cross-correlation is performed to get genuine homologous points. A density of 1 point per $\mathrm{km}^{2}$ is designed for SRP specification purposes. A subset of tie points is selected for bundle adjustment purposes (corresponding to 100 tie points for a small 60x60km SPOT 6/7 segment).

\subsubsection{Ground control point}

The usual case implies the use of the BDAmer database as GCP. BDAmer points are located directly in the SPOT 6/7 images thanks to the sensor PRIMARY level geometric model. Then, an image correlation is carried out between BDAmer and SPOT 6/7 images to keep only relevant GCP.

In some limited parts of the world, SPOT 5 HRS imagery (consequently BDAmer as well) may not be available. In this case, external GCP (as described in section 3.2.2) are measured directly on two SPOT 6/7 images and then correlated with all existing SPOT 6/7 images. The lack of the HRS imagery is compensated thanks to the addition of more GCP, more SPOT 6/7 strips, and by ICESat data that improve $\mathrm{Z}$ location and reliability.

3.5.3 SPOT 6/7 bundle block adjustment and quality
assessment

The software used to perform the bundle adjustment is fully described in (Massera and al., 2012). A typical check of the remaining equipment of the GCP and tie points (number, distribution, multiplicity and residual values) is achieved. Before SRP production, a full bundle adjustment quality assessment is performed thanks to Albatros data and other local data when available, not used as GCP in the bundle adjustment. An automatic method has been developed to assess planimetric differences between these control data and SPOT 6/7 orthoimages. Altimetry control is checked by a comparison between the tie point estimated elevation and a reference DSM (Albatros DSM or any other 3D relevant source).

The two figures below show examples of planimetric and altimetric quality check on the North America batch. Colored lines represent the differences between SRP and reference data (here, Albatros and USGS high resolution orthoimage). Obviously, in case of large residuals, further analysis is conducted to understand the reason for the differences. 


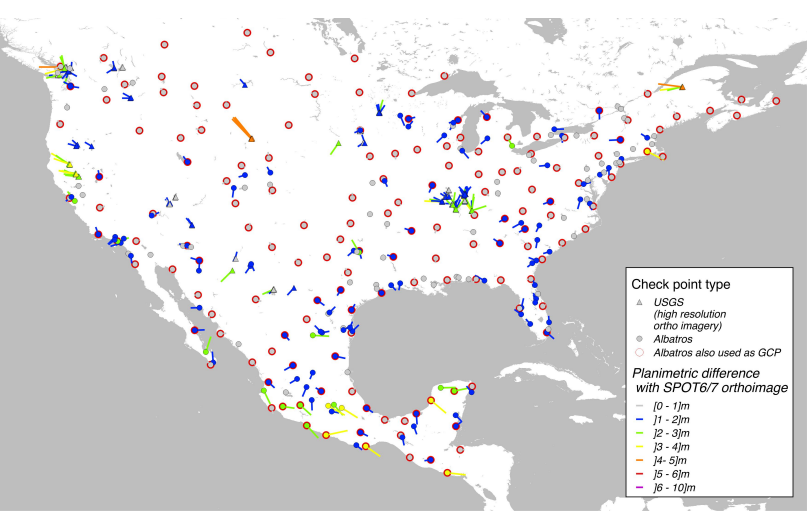

Figure 5. Planimetric check of SPOT 6/7 bundle adjustment

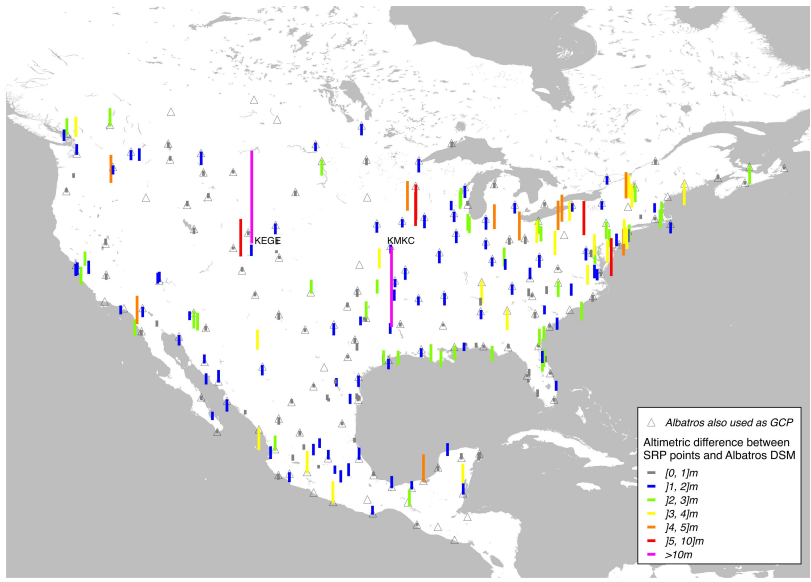

Figure 6. Altimetric check of SPOT 6/7 bundle adjustment KEGE and KMKC airports high altimetric differences are due to changes between Albatros Pléiades and SPOT 6/7 (airport duel tank), both data are correct

\subsection{SRP production}

\subsubsection{SRP generation and format delivering}

The final product is then built, if necessary, after a specific quality process, as presented in section 3.6.2.

Each GCP gathers one metadata file and an image chip set, GCP being at the centre.

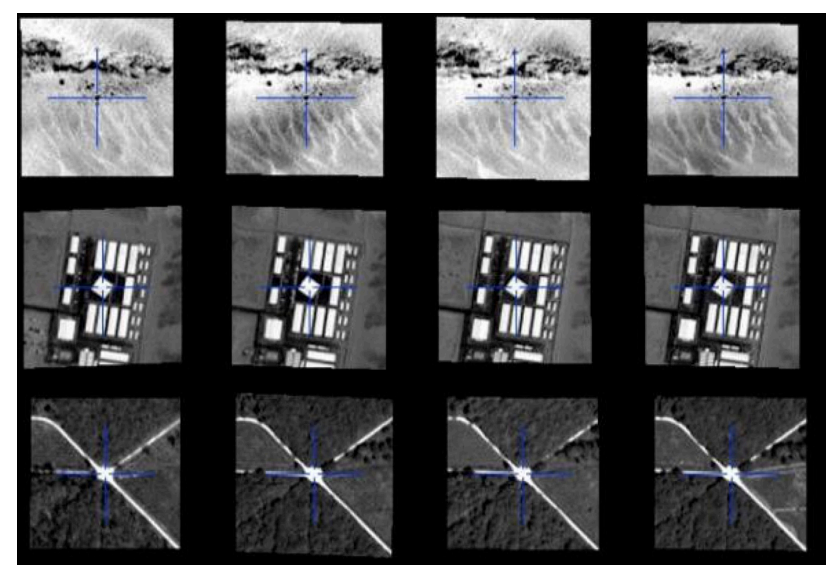

Figure 7. Examples of 3 GCP SRP points with 4 image chips

There are two types of image chips at a fixed size of 101x101 pixels:
A full resolution (2m) image chip set, upon a viewing area of $202 \times 202 \mathrm{~m}$ : one image chip is extracted from each qualified SPOT 6/7 measurement. These multiview and multi-date image chips are also able to handle seasonal effects on various regions.

A single overview image chip at subsampled resolution $(20 \mathrm{~m})$ : additionally, one image chip is extracted from the most representative image source by a 10 sub-sampling factor. The pixel chip size remaining the same, it enlarges the GCP viewing area of $2020 \times 2020 \mathrm{~m}$.

The image chips are extracts from SPOT 6/7 Panchromatic channel in reflectance integer count $\left(10^{4}\right.$ scale factor). They are rectified upon WGS84 at a constant ellipsoid altitude, mean value from all qualified stereo intersections on the GCP. The pixel resolution is normalized in degree unit according to the latitude, in order to avoid resampling artefacts. The GCP coordinates are those of the chip centre pixel. Image chips are formatted in JPEG 2000, lossless compression scheme, and encoded with GEOJP2 and GMLJP2 tags.

The GCP metadata file provides qualified information such as georeferencing, geometric accuracy, multiplicity, and lineage. Metadata are formatted in XML (ADS DIMAP format). The database is spatially organized in a tree data structure, partitioning the two-dimensional Earth space along parallels and meridians in geographic cells, recursively subdivided into 100 degrees, one-degree and 1/100 degree cells.

The precise matching of the GCP in a destination image is recommended with a consequent and relevant image chip set for multi-view observations. This will suppress outliers, refine the exact location, and offset off-nadir effects, resolution scaling and other deformations on the real shape of the feature in the destination image. For a fast registration, or to register destination image with poor native location, the matching may be initiated with the GCP overview single-view chip.

Perennial and freshness of the SRP database could be assumed by an update process, scanning the new acquisitions of the image satellite archive.

\subsubsection{Improvement for complex landscapes}

In some specific landscapes, a fraction of the feature points automatically extracted may represent non-perennial objects. When this phenomenon is detected (thanks to BDAmer experience or during quality check), specific processing is performed. Two user cases are described in this section.

\section{- Manual filtering on coastal regions}

Coastal regions are prone to unsuitable points such as specular effects on waves or moving boats. Thanks to a first automatic selection with water bodies' information, browsing is performed by operators in order to manually filter remaining errors.

\section{- $\quad$ Deep learning filtering on desert areas}

In the case of large deserts with sand dunes, detected feature points are often extracted on the dune peak or cavity (Figure 8, left, in red). Those points are not reliable since, in certain regions of the world, dunes may move up to 100 meters per year. 
The Rub' al Khali in the Arabian Peninsula is a sand desert which covers $650,000 \mathrm{~km}^{2}$. Former experience with HRS imagery and BDAmer database shows that in this case, many feature points occur on moving dunes. Yet, some good details can still be found: rock outcrop, plants described in (Ghazanfar and Fisher, 2013) or anthropic objects (see Figure 8, right, in green).
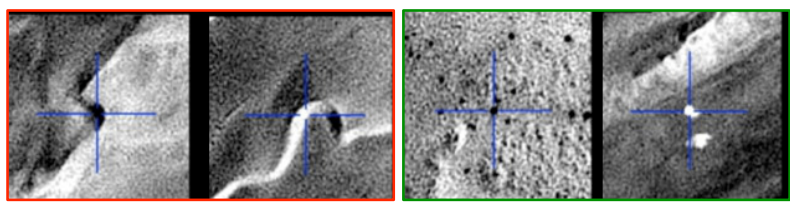

Figure 8. Feature points on desert: dunes (red) and other (green)

A method based on a deep learning classification algorithm has been developed to automatically detect dune points, and was applied on the Rub' al Khali desert. A training dataset of 5122 samples was built up manually (representing $1,8 \%$ of the entire dataset) and used in a typical convolutional neural network based on VGG16 (Simonyan, K., Zisserman, A, 2014). For each point, the algorithm provides a probability of belonging to a given class. To ensure the best reliability, the $9 \%$ of the points classified with a low confidence were shown to an operator for review. Quality control, conducted on a randomly selected subset of 25,084 points all over the area, shows $10 \%$ errors. This is a satisfying result, on par with human performance.

\section{PERFORMANCE ASSESSMENT}

\subsection{Proof of concept on Spain site}

In 2017, an early proof of concept was established on Spain. This country was chosen for its consequent size and various landscapes (urban, rural, mountainous, wild nature...). A prototype of first rough SRP database was used on 11 different test sites to refine on each site the 3D sensor model of Pléiades HR stereo images. The Spanish National Program for Aerial Orthophoto (PNOA) with an accuracy estimated at $1 \mathrm{~m} \mathrm{CE90,} \mathrm{see} \mathrm{(Villa,} \mathrm{2007),} \mathrm{was} \mathrm{used} \mathrm{as} \mathrm{independent}$ planimetric check points (see Table 3 ).

\begin{tabular}{|l|l|c|}
\hline Test site & Landscape & $\begin{array}{c}\text { Check points XY } \\
\text { residues (CE90, m) }\end{array}$ \\
\hline Alcaniz & Barren landscape & 3.0 \\
\hline Aragon & Barren landscape & 3.0 \\
\hline Asturias & Medium mountain, dense vegetation & 1.6 \\
\hline Barcelona & Urban, coast, hilly & 3.0 \\
\hline Estremadure & Field crops & 3.0 \\
\hline Huesa & Medium mountain, poor vegetation & 3.6 \\
\hline Madrid & Urban & 2.6 \\
\hline Salamanque & Field crops & 2.6 \\
\hline Santander & Urban, coast, hilly & 1.7 \\
\hline Valladolid & Urban & 2.3 \\
\hline Valencia & Urban, crop fields & 2.1 \\
\hline
\end{tabular}

Table 3. Accuracy assessment on Spain test sites

Results on this SRP prototype were around 3mCE90, demonstrating such level of specification could be targeted for a final SRP product.

\subsection{Real performance assessment on China}

In 2019, a campaign was performed to assess the planimetric performance target of 5m CE90 for standard orthoimagery: the sensor model was first adjusted on SRP GCP and then rectified with WorldDEM4Ortho (WD4O). The SRP final product and WD4O V2 data over China were used.
WD4O V2 is a hybrid DTM/DSM derived from the WorldDEM DSM which fulfills Level 1 of the NGA High Resolution Elevation Product specification (NGA, 2020). WD4O have an absolute vertical accuracy specification of $<4$ m LE90 and a relative specification of $<2$ m LE90 on slopes $<20 \%$.

At first, the global quality of elevation values from SRP points toward WD4O was assessed. The absolute difference between WD4O and SRP elevation values was computed on areas supposed to share a similar on-ground reference surface. The results were statistically agglomerated, and elevation RMS was computed at degree size square cell (see Figure 9). Because of differences of covering along the China borders and shorelines, statistics may not be reliable and some artefacts may occur.

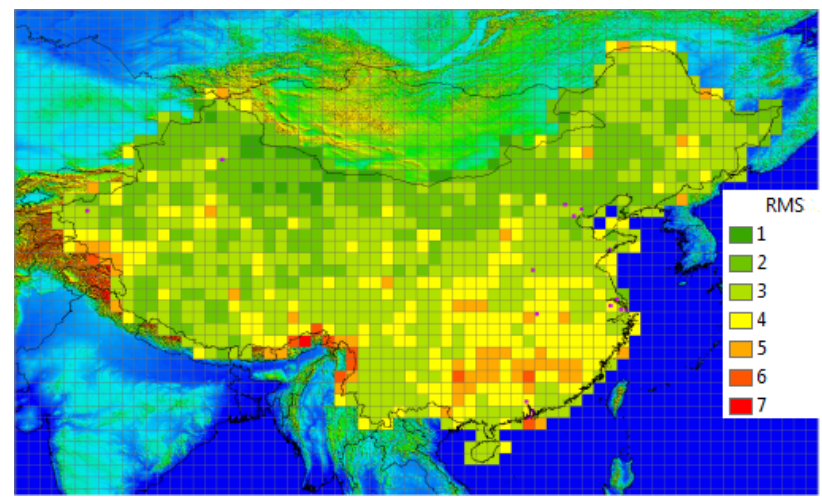

Figure 9. China, RMS elevation difference $(\mathrm{m})$ between SRP points and WD4O V2 DTM/DSM

Most measurements range between 1 and $4 \mathrm{~m}$, denoting a good consistency and rigidity of SRP data. Higher differences in the south-east part of China may be explained by large forested areas where DSM and SRP may represent the reference surface differently. Future works are on-going to deeper demonstrate the SRP elevation quality including with ICESat-2 data.

Secondly, the refined image planimetric accuracy has been assessed on 8 different sites using Pléiades $1 \mathrm{~A} / 1 \mathrm{~B} 50 \mathrm{~cm}$ single image (see Table 4). Accuracy of both check sources is $1 \mathrm{~m}$ CE90 or better.

\begin{tabular}{|l|l|c|c|c|}
\hline Test site & \multicolumn{1}{|c|}{ Landscape } & $\begin{array}{c}\text { Pléiades } \\
\text { incidence } \\
\text { angle } \\
\text { (degree) }\end{array}$ & $\begin{array}{c}\text { Check } \\
\text { points XY } \\
\text { residues } \\
\text { (CE90, m) }\end{array}$ & $\begin{array}{c}\text { Ortho quality } \\
\text { check } \\
\text { (absolute } \\
\text { CE90, m) }\end{array}$ \\
\hline Wuhan & Rural, water areas & 13.8 & 2.2 & 2.5 \\
\hline Kashgar & $\begin{array}{l}\text { Rural, semi-desert } \\
\text { moderate mountain }\end{array}$ & 15.8 & 3.2 & 2.4 \\
\hline Shenzhen & Urban, mountain & 16.0 & 2.0 & 2.8 \\
\hline Beijing & Urban, highlands & 16.8 & 1.5 & 1.8 \\
\hline Macau & $\begin{array}{l}\text { Urban, isolated } \\
\text { mountain }\end{array}$ & 18.2 & 1.7 & 4.1 \\
\hline Urumqi & $\begin{array}{l}\text { Rural, semi-desert } \\
\text { mountain }\end{array}$ & 20.1 & 1.6 & 1.8 \\
\hline Zhengzhou & Rural & 19.7 & 3.0 & 2.2 \\
\hline Shanghai & Urban, highlands & 22.6 & 2.5 & 3.9 \\
\hline
\end{tabular}

Table 4. Accuracy assessment on China test sites

Independent control points are introduced to check the bundle adjustment accuracy (XY residues). Airbus Albatros airport orthoimages (see section 3.2.2) are used to check the final orthoimages rectified with WD4O. The $5 \mathrm{~m}$ CE90 planimetric accuracy for single orthoimagery is largely achieved. These first results will be consolidated in the future. 


\subsection{Performance of SRP for images with poor native location}

As explained above, every SRP point goes with one subsampled overview chip in order to handle destination images with poor native image location. An experiment was carried out on two different datasets to validate this concept. On these test sites, a direct SRP registration of Pléiades images was performed and compared with:

- Simulated poor location (0): attitudes were moved randomly in yaw/pitch/roll, thus initial location moved by approximately one or a few hundred meters.

- Coarse registration (1): images are registered on the SRP only with the subsampled chips.

- Fine registration (2): images are registered with the fullresolution chips. It is expected for this location to be close to the direct location.

The table below shows that similar results can be reached for the two datasets on Spain.

\begin{tabular}{|c|c|c|c|c|c|c|}
\hline Differences with SRP & \multicolumn{2}{|c|}{ Valencia } & \multicolumn{2}{c|}{ Galicia - test1 } & \multicolumn{2}{c|}{ Galicia - test2 } \\
\cline { 2 - 7 } registration (meter) & $\mathbf{d X}$ & $\mathbf{d Y}$ & $\mathbf{d X}$ & $\mathbf{d Y}$ & $\mathbf{d X}$ & $\mathbf{d Y}$ \\
\hline $\begin{array}{c}\text { 0. Simulated poor } \\
\text { location }\end{array}$ & -130.9 & 101.0 & -123.3 & 184.0 & -204.2 & 216.3 \\
\hline $\begin{array}{c}\text { 1. Coarse registration } \\
\text { (overview single-view } \\
\text { chips) }\end{array}$ & -0.6 & -1.3 & -0.1 & 0.7 & -0.8 & -3.4 \\
\hline $\begin{array}{c}\text { 2. Fine registration } \\
\text { (full resolution multi- } \\
\text { view chips) }\end{array}$ & $\mathbf{0 . 1}$ & $\mathbf{0 . 0}$ & $\mathbf{0 . 1}$ & $\mathbf{0 . 0}$ & $\mathbf{0 . 1}$ & $\mathbf{- 0 . 4}$ \\
\hline
\end{tabular}

Table 5. Performance of SRP for images with poor native location

\section{ON-GOING PRODUCTION AND CONCLUSION}

\subsection{Results at mid-term project}

The complete workflow is operational since March 2018. More than 47Mkm2 (Europe \& Turkey, West Russia \& Middle East, North America \& Mexico, East Continental Asia) were produced from the beginning of the project in March 2018, to September 2019. 57Mkm2 (Africa, Australia and South and Central America) are currently in progress (February 2020).

On the $47 \mathrm{Mkm}^{2}$ already produced:

- GCP density reached satisfies the expected specifications as shown in Figure 11: $91.7 \%$ of 1/100 degree geocell fulfil the 1 point per $2 \mathrm{~km}^{2}$ specification. As expected, density is sometimes relaxed in typical difficult landscapes (snow, sandy desert, areas with persistent cloud,...).

- GCP accuracy is also in accordance with the $3 \mathrm{~m}$ CE90 specification since it has been estimated with Albatros data at $2.6 \mathrm{~m} \mathrm{CE90.}$

On the $57 \mathrm{Mkm}^{2}$ currently in progress, work has been carried out to improve SRP density on frequently cloudy regions (tropical climate), see section 3.2, "relaxed cloud cover constraint".

\subsection{Conclusion}

In January 2020, the Space Reference Point workflow has a production background of nearly $100 \mathrm{Mkm}^{2}$. The next batches on high latitudes (low anthropic, snow cover), scattered and isolated islands would probably require new optimizations. Work is already in progress to reach the best possible accuracy.

With benefits from twenty years of common work and experience shared between IGN and ADS, the SRP database offers a worldwide, accurate and straightforward registration service for various images. Such a service can target a location accuracy of $3 \mathrm{~m}$ for the sensor model and a horizontal performance of $5 \mathrm{~m}$ for orthoimages in a massive standard process. SRP is an evolving concept that can easily be upgraded with new images, ensuring a potential infinite up-to-dateness.

\section{REFERENCES}

Airbus, 2020. Pléiades Neo Trusted Intelligence. https://www.intelligence-airbusds.com/en/8671-pleiades-neotrusted-intelligence (23 January 2020).

Airbus, 2020-b

WorldDEM4Ortho

https://www.airbus.com/newsroom/press-

releases/en/2017/07/airbus-releases-worlddem4ortho--the-mostaccurate-elevation-mode.html (23 January 2020).

Bouillon A., Bernard M., Gigord P., Orsoni A., Rudowski V., Baudoin A., 2006. SPOT 5 geometric performances: Using block adjustment as a key issue to improve quality of DEM generation. ISPRS Journal of Photogrammetry \& Remote Sensing 60 (2006) 134-136.

Breton, E., Orsoni, A., Lasselin, D., Pausader, M., 2009. Ready to use solution to give a boost to African countries' base maps. IGN, Institut Géographique National. Africa GIS Conference, Kampala, Uganda 2009.

Gascon, F., Bouzinac, C., Thépaut, O., Jung, M., Francesconi, B. et al., 2017. Copernicus Sentinel-2A Calibration and Products Validation Status. Remote Sensing, MDPI, 2017, 9 (6), pp.1-81. 10.3390/rs9060584. Hal-01853316

Ghazanfar S.A, Fisher, M., 2013 : Vegetation of the Arabian Peninsula, Springer Science \& Business Media

Massera, S., Favé, P., Gachet, R., Orsoni, A., 2012. Toward a global bundle adjustment of SPOT 5 - HRS Images. Int. Arch. Photogramm. Remote Sens. Spatial Inf. Sci., XXXIX-B1, 251256, doi.org/10.5194/isprsarchives-XXXIX-B1-251-2012.

NGA, 2009. Implementation Profile for High Resolution Elevation (HRE) Products. Version 1.0 https://nsgreg.nga.mil/NSGDOC/files/doc/Document/NGA.IP.0 002_1.0\%20HRE.pdf, (23 January 2020)

Simonyan, K. and Zisserman, A., 2014. Very Deep Convolutional Networks for Large-Scale Image Recognition. arXiv preprintarXiv: 1409.1556.

SPOT Image, 2011. Reference3D Product Description. Version 6.1. https://www.intelligenceairbusds.com/files/pmedia/public/r469 9 reference3d product description_201105.pdf, (23 January 2020 )

Villa, G., 2007. PNOA (Spanish National Program for Aerial Orthophoto): collaborative and innovative spirit in Spanish geospatial information. Geoinformatics. 


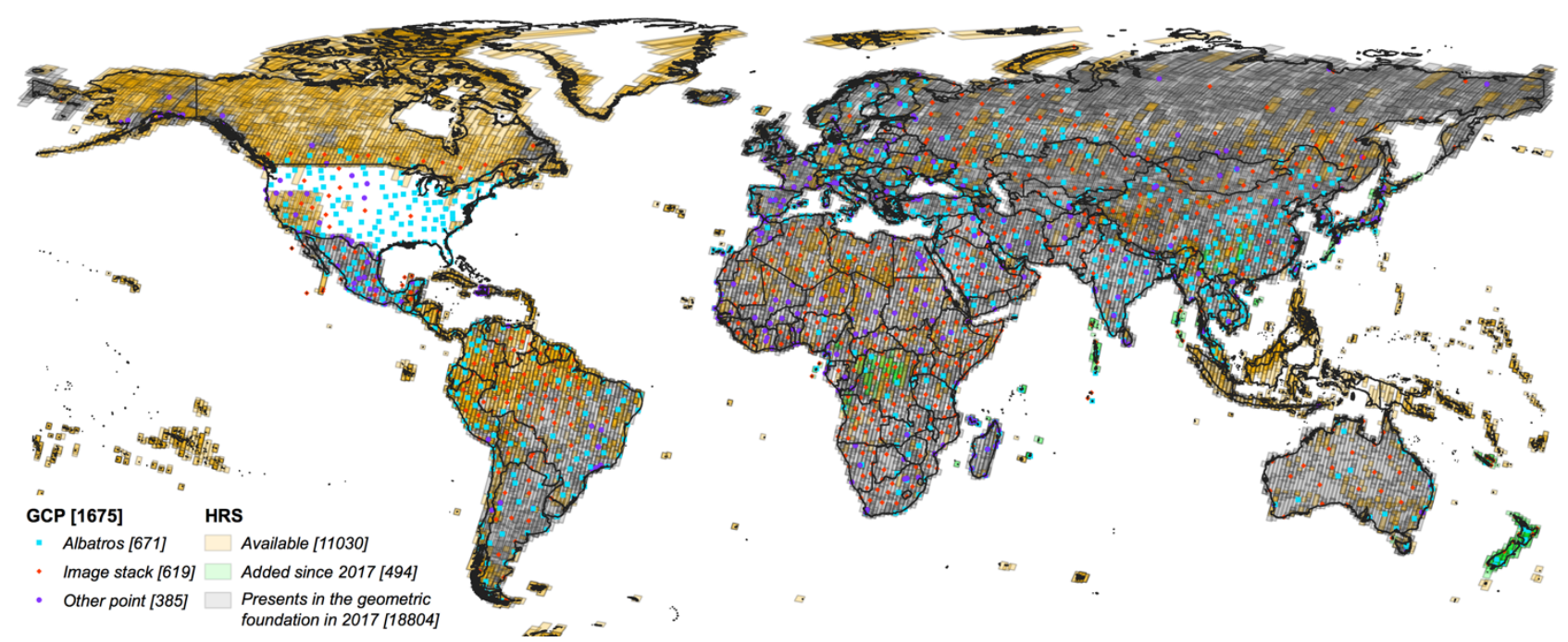

Figure 10. Geometric foundation - January, 2020

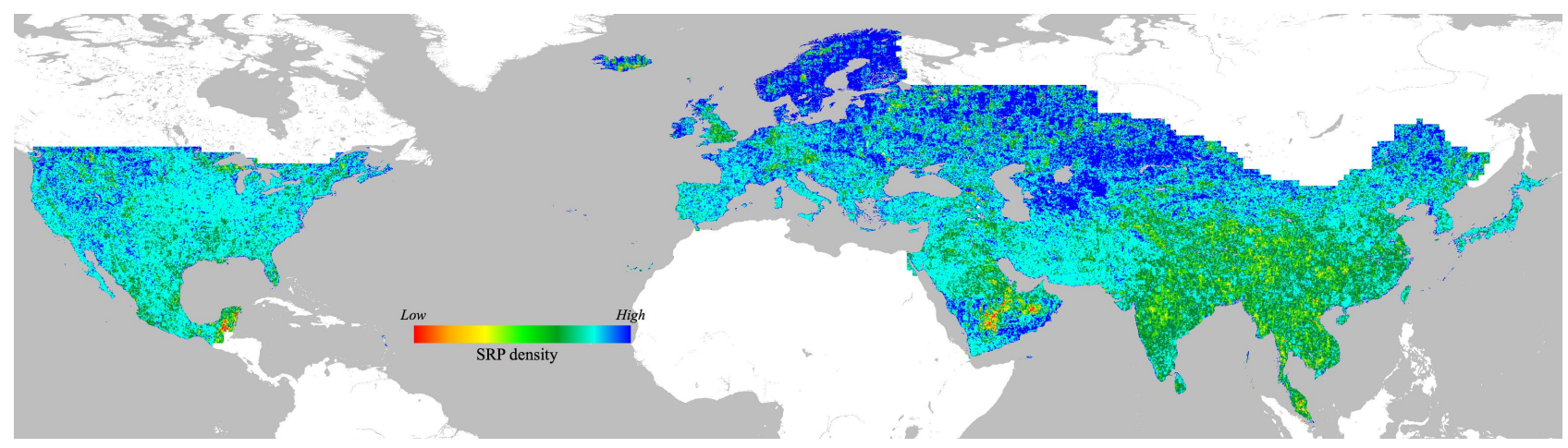

Figure 11. SRP density status - September, 2019 